\title{
Quasi-lattices of qubits for generating inequivalent multipartite entanglements
}

\author{
Hou $\operatorname{Ian}^{1}$ \\ ${ }^{1}$ Institute of Applied Physics and Materials Engineering, FST, University of Macau, Macau
}

\begin{abstract}
The mesoscopic scale of superconducting qubits makes their inter-spacings comparable to the scale of wavelength of a circuit cavity field to which they commonly couple. This comparability results in inhomogeneous coupling strengthes for each qubit and hence asynchronous Rabi excitation cycles among the qubits that form a quasi-lattice. We find that such inhomogeneous coupling benefits the formation of multi-photon resonances between the single-mode cavity field and the quasi-lattice. The multi-photon resonances lead, in turn, to the simultaneous generation of inequivalent $|\mathrm{GHZ}\rangle$ and $|W\rangle$ types of multipartite entanglement states, which are not transformable to each other through local operations with classical communications. Applying the model on the 3-qubit quasilattice and using the entanglement measures of both concurrence and 3-tangle, we verify that the inhomogeneous coupling specifically promotes the generation of the totally inseparable $|\mathrm{GHZ}\rangle$ state.
\end{abstract}

\section{INTRODUCTION}

Superconducting qubits are artificial two-level atoms made from superconducting islands linked by multiple Josephson junctions [1]. When coupled to a microwave field via a transmission line or coplanar waveguide resonator in the circuits, they become a circuit quantum electrodynamic (QED) system [2]. Circuit QED is equivalent to cavity QED for natural atoms and circuit versions of optical effects [3], such as lasing [4, 5], electromagnetically induced transparency [ 6 [ 8 ], and parametric amplification [9], have already been demonstrated.

Over the years, the studies on circuit QED have been extended to multi-qubit systems [10, 11] and their entanglement property [12], superradiant states [13, 14] as well as phase transitions [15] have been experimentally explored. In the fabricated circuits, these artificial atoms are orders of magnitude (usually $10^{3}$ to $10^{4}$ in linear scale) larger than their natural counterparts. To make existing theories and models for optical cavities relevant to circuit systems, the experimental remedies are: (i) placing the qubits exactly at the antinodes of the resonator mode [10] so that Tavis-Cummings model becomes applicable; (ii) by stretching the transmission line such that the qubits are congregated at the center [11] so that the Dicke model adopted in optomechanical system [16 18] becomes applicable; or (iii) by fabricating dedicated resonators for each qubit and externally synchronizing the drivings to each resonator [19]. All of these approaches effectively reduce the multi-qubit systems back to conventional scenarios one meets in optical cavities. However, under a general scenario, the interspacing between two neighborings qubits is comparable with the resonator mode wavelength and the qubits do not necessarily lie on the anti-nodes, letting the qubitresonator coupling become inhomogeneous and rendering conventional theoretical models irrelevant for multi-qubit circuits.

Here, we consider a general multi-qubit system as a quasi-lattice: a lattice that has each artificial atom located at a fixed lattice point and two neighboring artificial atoms distanced by a fixed lattice spacing, though each artificial atom receives a waveform-dependent coupling strength to a single-mode cavity field. That means, we disregard the Dicke model that only considers the scaling effect of coupling and consider rather the Rabi frequency of each qubit to be uneven such that the excitations throughout the quasi-lattice are asynchronous. Based on the projected deformed SU(2) algebra method [20], we theorize the quasi-lattice as a large collective spin that regard the lattice-resonator interaction as a quasi-particle excitation. The quasi-particle states are a set of multi-photon stationary resonant states that diagonalize the interaction Hamiltonian. Then, by employing the entanglement measures of concurrence [21, 22] and 3-tangle 23] on these states, we determine that both the $|\mathrm{GHZ}\rangle$ and the $|W\rangle$ entangled states are cogenerated simultaneously during the resonant processes. In particular, with the quasi-lattice being asynchronously excited, the generation of $|\mathrm{GHZ}\rangle$ state is optimized over past homogeneous-coupling schemes [24, 25]. By linking the asynchronicity statically to a deformation factor of the underlying algebra, one can associate maximized entanglement measures that characterizes the $|\mathrm{GHZ}\rangle$ state at specific scenarios of asynchronous excitation. The entanglement optimization was proved earlier to be controllable via the coupling strength when two qubit are directly capacitatively coupled to each other [26].

Since the $W$ and the GHZ states are inequivalent to each other [27, 28], i.e. one cannot obtain one state from the other through local operations with classical communications (LOCC), the ability to generate both in a unified system greatly reduces our reliance on complex quantum gate routines currently employed [19]. Especially, the difficulty in generating GHZ-type entanglement shows the potential of the quasi-lattice structure as a fundamental building block of future quantum information processing devices. This peculiar feature of the quasi-lattice can be attributed, following Dicke's notions, to its avoidance of full population inversion because of the asynchronous excitations and thus yielding a higher cooperation among the qubits. Consequently, the quasilattice receives higher probability in absorbing multiple 


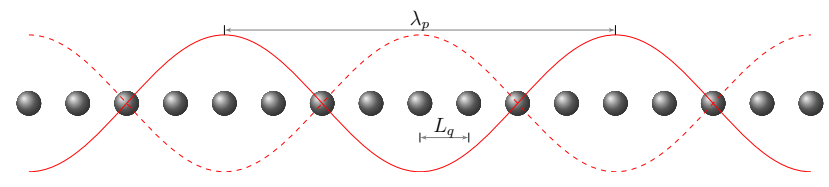

FIG. 1. (color online) Illustrations of inhomogeneous interactions on a quasi-lattice. Uniform lattice spacing $L_{\mathrm{q}}$ between two neighboring qubits is on the same order of magnitude as the wavelength $\lambda_{\mathrm{p}}$ of the transmission line cavity resonator. Their ratio $\ell$ determines the quasi-particle excitation on the lattice. The illustrated quasi-lattice with 17 qubits has $\ell=1 / 4$.

photons from the resonator, resulting in a more entangled state than it would be achievable with homogeneous coupling. Moreover, it was shown that the mechanism to generate $W$-states becomes faster when the coupling is inhomogeneous [29], which verifies from another angle that the inhomogeneous coupling of quasi-lattices facilitates entanglement generation.

\section{QUASI-LATTICE}

To identify the problem more clearly, we model the system on the time-independent Hamiltonian $(\hbar=1)$

$$
H=\omega_{0} a^{\dagger} a+\sum_{j=0}^{N-1}\left[\omega_{\mathrm{q}} \sigma_{j, z}+g_{j}\left(\sigma_{j,+} a+\sigma_{j,-} a^{\dagger}\right)\right] .
$$

Under the formulation of circuit QED [2], each superconducting qubit is approximated by a two-level system whose eigenenergy $\omega_{\mathrm{q}}=\sqrt{E_{C}^{2}+E_{J}^{2}}$ is diagonalized from the tunable charge energy $E_{C}$ and junction energy $E_{J}$ of the Josephson junctions. We denote the qubit at the $j$-th position by the Pauli matrix $\sigma_{j, z}$ and the singlemode transmission line resonator by the pair of creation and annihilation operators $a^{\dagger}$ and $a$. We consider all $N$ qubits be tuned uniform at the eigenfrequency $\omega_{\mathrm{q}}$ that is closed resonant to the resonator frequency $\omega_{0}$. Assuming $N$ is not sufficiently large on the mesoscopic scale to warrant collective effects on the virtual photon processes, the interaction between each qubit and the resonator is a Jaynes-Cummings type dipole-field coupling, where the non-rotating terms are discarded.

The lattice spacing $L_{\mathrm{q}}$, the distance between two neighboring qubits, is assumed uniform throughout the quasi-lattice. Since we consider $L_{\mathrm{q}}$ to be comparable to the wavelength $\lambda_{\mathrm{p}}$ of the resonator mode, we find a sinusoidal dependence of the coupling strength $g_{j}$ on the discrete position $j$ relative to the waveform shape of the standing cavity mode: $g_{j}=g \cos (j \pi \ell)$. In this inhomogeneous coupling, $\ell=2 L_{\mathrm{q}} / \lambda_{\mathrm{p}}$ is the dimensionless reduced qubit spacing with respect to the wavelength $\lambda_{\mathrm{p}}$, where the factor 2 is added to simplify the derivations given below.

Illustrated in Fig. 1] we can regard $\ell$ as the relative lattice spacing and $j$ as the dimensionless integer coordinate in units of $L_{\mathrm{q}}$ for the one-dimensional quasi-lattice. One can see that different sets of qubits will be driven at different Rabi frequencies by either variably spacing the qubits or controlling the harmonic modes in the transmission line resonator [30]. If each qubit is driven at the same rate by the resonator, neighboring qubits will become fully excited in a synchronous fashion. The spontaneous radiation rate of each individual qubit reaches maximum at the same rate. Consequently, the probability of simultaneous absorption of multiple photons is low. In contrast, if the quasi-lattice has inhomogeneous coupling to the resonator mode, neighboring quits will become fully excited at asynchronous Rabi frequencies. That means while some qubit is spontaneously emitting photons, others which are not fully excited not only absorb the resonator photons but also have high probability to reabsorb the emitted photons from neighboring qubits. This circumstance leads to a high probability of multiphoton lattice-cavity resonance.

\section{QUASI-PARTICLES}

To verify that the inhomogeneous coupling indeed increases the probability of multi-photon resonance in the qubits, we examine the eigenspectrum of the collective quasi-particle excitations in the quasi-lattice. For such collective excitations, each quantized energy level does not correspond to the energy level of the excited state of a particular qubit, but to a delocalized exciton with different levels of excitation probability distributed across the quasi-lattice as a whole.

To give analytical expressions for the eigenstates of the quasi-particles, we normalize the inhomogeneous coupling to the nominal maximum coupling rate $g$ and incorporate the inhomogeneous factors into the ladder operators of a large collective spin. That is, we write the interaction part of Eq. (1) as $H_{\text {int }}=g\left(S_{+} a+S_{-} a^{\dagger}\right)$ where $S_{ \pm}=\sum_{j} \cos (j \pi \ell) \sigma_{j, \pm}$. Correspondingly, the free Hamiltonian can be written as $H_{0}=\omega_{\mathrm{q}} S_{z}+\omega_{0} a^{\dagger} a$, where $S_{z}=\sum_{j} \sigma_{j, z}$ accounts for the spin magnetic moment. The quasi-particle states are therefore the resonant states between the resonator photons and the qubit excitations, which diagonalize the Hamiltonian $H=H_{0}+H_{\text {int }}$ in the collective spin formulation.

In order to make the interaction Hamiltonian blockdiagonalizable like that of Tavis-Cummings (TC) model, the set of operators $\left\{S_{ \pm}, S_{z}\right\}$ must conform to a $\mathrm{SU}(2)$ Lie algebra. Nonetheless, since $\left[S_{+}, S_{-}\right] \neq 2 S_{z}$ because of the non-uniform coupling in the quasi-lattice, we approximate the set as a deformed algebra by projecting the commutator into the $\mathrm{SU}(2)$ group manifold through a trace over the Hilbert space spanned by the quasi-lattice. Geometrically, this method associates with the deformation of the Bloch sphere $\mathbb{S}^{2}$, the two-dimensional projection from the 3 -sphere $\mathbb{S}^{3}$ that is isomorphic to $\mathrm{SU}(2)$, into an ellipsoid [20, 30]. The degree of elongation of the 
resulting ellipsoid from the original 2-sphere is captured by the c-number deformation factor $\mathfrak{f}$ in the commutation relation $\left[S_{+}, S_{-}\right]=2 \mathfrak{f} S_{z}$.

Algebraically, the deformation factor $\mathfrak{f}$ measures how the new spin operators $\left\{S_{ \pm}, S_{z}\right\}$ differ from their counterparts in a standard $\mathrm{SU}(2)$ algebra. In other words, as a function as $\ell$ and $N$, it figuratively quantifies the degree the quasi-lattice resembles a normal lattice. Its value to the first-order approximation under the Hilbert-Schmidt norm is $\mathfrak{f}=\sum_{j} g_{j}^{2} / N$. With $g_{j}$ being the cosines defined above, we have

$$
\mathfrak{f}=\frac{1}{2}+\frac{1}{4 N}\left[1+\frac{\sin (2 N-1) \pi \ell}{\sin \pi \ell}\right] .
$$

When the qubits are inhomogeneously distributed and thus asynchronously driven at uneven rates, $\ell$ takes a fractional value and $\mathfrak{f}$ adopts a positive sub-unit value. On the other hand, when (all qubits fall on the antinodes on the resonator mode) or the limiting value of zero (the inter-qubit spacing is negligible compared to the resonator wavelength, as in a natural lattice), $\ell$ take an integer value and $\mathfrak{f}$ reduces to one, signifying the quasi-lattice falls back to a homogeneous lattice structure.

After the projection-deformation process described above, the Hamiltonian in Eq. (11) can be diagonalized in the usual angular momentum states under TC-model, the eigenvalues of which read

$$
E_{u}=\omega_{\mathrm{q}} u+\varepsilon
$$

for a quasi-particle "excitation" number $u=n+m$, which is distributed among the photon count $n$ from the resonator and the spin moment $m$ from the quasi-lattice. Since we have adopted the angular momentum model, $u$ can take either integer or half-integer values greater than $(-N / 2)$. The second term $\varepsilon$ is the energy splitting that accounts for the qubit-photon detuning $\Delta \omega=\omega_{0}-\omega_{\mathrm{q}}$ and the lattice-photon interaction with maximum coupling strength $g$ introduced. At resonance $\Delta \omega=0, \varepsilon$ would become a multiple of $g$ only. In brief, the resonator mode dresses the quasi-lattice states into a series of clusters of states, grouped by the value of $u$.

Given the eigenenergy in Eq. (3) for each $u$, one can associate a time-independent Schroedinger equation with the eigenvector

$$
|u, r\rangle=\sum_{n=0}^{u+r} c_{n}|n\rangle \otimes|r, m\rangle,
$$

which is parametrized additionally by the total spin number $r$ for $N$ qubits. It has a $(u+r+1)$-fold degeneracy distributed among different combinations of $n$ and $m$, which is split by the finite interaction into finer levels with splitting $\varepsilon$, analogous to ac-Stark shifts to an atom. Solving the time-independent eigen-equation $H|u, r\rangle=E_{u}|u, r\rangle$ using Eq. (3)-(44), one finds that both this splitting $\varepsilon$ and the coefficients $c_{n}$ are determined a posteriori by a recursive relation

$$
c_{n}=\frac{\varepsilon-\Delta \omega(n-1)}{g \alpha_{r, m} \sqrt{n f}} c_{n-1}-\sqrt{\frac{n-1}{n}} \frac{\alpha_{r, m+1}}{\alpha_{r, m}} c_{n-2}
$$

with initial conditions $c_{u+r+1}=c_{-1}=0$. In the relation, $\alpha_{r, m}=\sqrt{(r-m)(r+m+1)}$ are the off-diagonal elements of the ladder operators. The initial value $c_{0}$ can take arbitrary value since the subsequent coefficients are all multiples of $c_{0}$, hence it can be regarded as a constant arbitrarily chosen for normalization. The analytical expressions for $c_{n}$ and $\varepsilon$ can be found by treating Eq. (5) as a difference equation [20]. The coefficients $c_{n}$, which represent the probability amplitudes of different mixed resonant states between the quasi-lattice and the resonator photon, are not only determined by the offdiagonal transition elements, but are also determined by the deformation factor $\mathfrak{f}$. The latter is thus a structure parameter of the quasi-lattice that decides the form each photon is transferred to all the qubits, affecting how entanglement is generated among the qubits.

Dynamically speaking, the quasi-particle states described by Eq. (4) are the stationary state of the latticecavity system when the qubits are collectively resonant with the electromagnetic cavity mode. They also serve as a set of transformed or dressed bases for the combined system, which is parametrized by $u$ and $r$ after the transformation. Conversely, any initial states given as a tensor product of an arbitrary cavity Fock states $\sum_{n} d_{n}|n\rangle$ (e.g. $d_{n}$ could be $\alpha^{n} / \sqrt{n !}$ for a coherent state) and the lattice angular momentum state $|r, m\rangle$ can be decomposed in the dressed bases as

$$
|\psi\rangle=\sum_{n} d_{n}|n\rangle \otimes|r, m\rangle=\sum_{u} \delta_{u}|u, r\rangle .
$$

Then, by regarding each sequence $\left\{c_{n}\right\},\left\{d_{n}\right\}$, and $\left\{\delta_{n}\right\}$ as the column matrix $\mathbf{c}$, d, and, respectively, one can form the transformation matrix $[C]_{\text {un }}$ from Eq. (44) to find $\delta=C^{-1} \mathbf{d}$.

\section{MULTI-PHOTON RESONANCES}

The coefficients of each resonant stationary state, as determined by Eq. (5), shows that the resonant state is unequally contributed by a set of quasi-lattice configurations $|r, m\rangle$. If the quasi-lattice is initialized to the ground configuration $|r,-r\rangle$, then different numbers of photons are brought into resonance with the quasi-lattice to form the resulting superposition state with different $m$. In the case of superconducting quantum circuits, this is achieved by sending pulse signals of different $\pi$ lengths into the qubits whose level spacings are tuned in-resonance with the waveguide resonator [19]. In the following, a particular eigenstate $|u, r\rangle$ with a fixed $u$ is considered. 


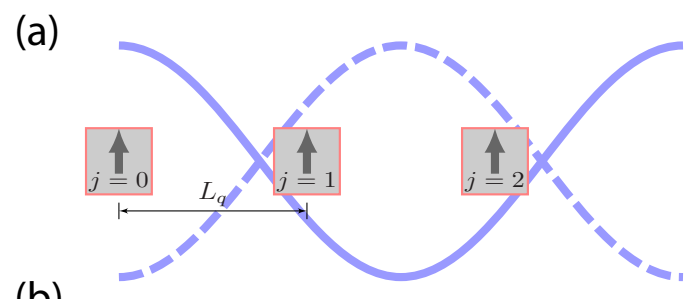

(b)

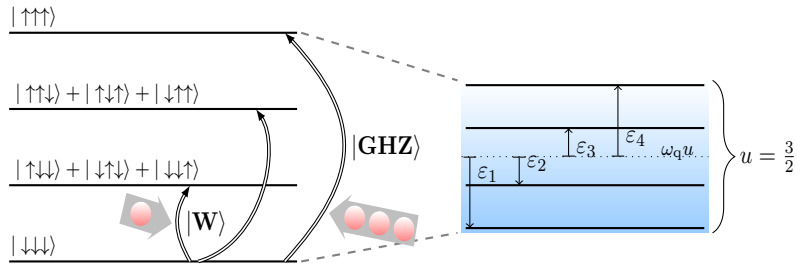

FIG. 2. (color online) (a) Illustration of a 3-qubit quasi-lattice with $\ell=2 / 3$. (b) The level diagram of the 3 -qubit quasilattice, with the ground state resonant with multiple photons, forming two classes of entanglement states $(|\mathrm{GHZ}\rangle$ and $|W\rangle$ states). The energy levels are effectively dressed into a cluster of states of the same "excitation" number $3 / 2$, split by shifts $\varepsilon_{1,2,3,4}$ due to the lattice-photon interaction.

In a resonant process, the excitation number $u$ shared between the photon Fock states and the quasi-lattice angular-momentum states is conserved. The fixed $u$ permits both single-photon resonance (between $|n=u+r\rangle$ and $|n=u+r-1\rangle)$ and multi-photon resonances (between $|n=u+r\rangle$ and $|n=u-m\rangle$ where $m>-r+1)$. In addition, the inhomogeneous coupling quantified by the deformation factor $\mathfrak{f}$ plays a part in Eq. (5) for the multi-photon processes. To investigate what effect it can exert, we look at the patterns of entanglements formed on the quasi-lattice through its variation range and discern, specifically, how these patterns associate with the two entanglement types of $|\mathrm{GHZ}\rangle$ state and $|W\rangle$ state.

For a $N$-qubit quasi-lattice whence $r=N / 2$, the $|\mathrm{GHZ}\rangle$ state is generated from an $N$-photon resonance between the lattice states $|r,-r\rangle$ and $|r, r\rangle$. For a quasiparticle excitation with excitation number $u$, it constitutes the two terms $c_{N}|N\rangle \otimes|r,-r\rangle+c_{0}|0\rangle \otimes|r, r\rangle$ within the decomposition of $|u, r\rangle$ for any $u$. Whereas, the $|W\rangle$ state is generated from an one-photon resonance, i.e. it associates with the terms $c_{u+r}|u+r\rangle \otimes|r,-r\rangle+$ $c_{u+r-1}|u+r-1\rangle \otimes|r,-r+1\rangle$, depending on a particular $u$ for the total system excitation. (If the inverted $|\bar{W}\rangle$ state is also considered, the term $c_{u-r+1}|r, r-1\rangle$ should be included.) The general consideration is therefore whether the distribution of the coefficients $\left\{c_{n}\right\}$ as determined by Eq. (5) would concentrate on $c_{0}$ and $c_{u-r+1}$ (and $c_{u-r+1}$ ) if the deformation factor $\mathfrak{f}$ takes value away from either 0 or 1 , indicating an inhomogeneous coupling and thus asynchronous excitation scheme.

Since solving Eq. (5) for an expression of $c_{n}$ would not illustrate clearly the change in the distribution and, more importantly, researches on general $N$-qubit entanglement measures are plural with no particular established ones, we examine a simplified case of a 3-qubit quasi-lattice for further considerations. The 3-qubit system has two metrics of entanglement that can distinctively discriminate a $|\mathrm{GHZ}\rangle$ state from a $|W\rangle$ state. The model is illustrated in Fig. 2(a) and the resonant processes are illustrated in Fig. 2 (b) for the case of $u=\frac{3}{2}$, for which the clustered dressed level is split into four sublevels for the four values of the splitting $\varepsilon$. The quasi-lattice at ground state $\left|\frac{3}{2},-\frac{3}{2}\right\rangle$ can be resonant with both $\left|\frac{3}{2}, \frac{3}{2}\right\rangle$ through a triple-photon process to generate the $|\mathrm{GHZ}\rangle$ state and $\left|\frac{3}{2},-\frac{1}{2}\right\rangle$ through a single-photon process to generate the $|W\rangle$ state, as shown on the left side of Fig. 2(a). The state $\left|\frac{3}{2}, \frac{1}{2}\right\rangle$ is the inverted $|\bar{W}\rangle$ state, which is equivalent to $\left|\frac{3}{2},-\frac{1}{2}\right\rangle$ up to an LOCC operation and can be generated from the quasi-lattice ground state through a double-photon resonance.

To see how asynchronous excitations benefit the generation of $|\mathrm{GHZ}\rangle$ type of entanglement in a stationary state, we calculate two coefficients $c_{0}$ and $c_{3}$ for $\left|u=\frac{3}{2}\right\rangle$, which associate respectively with the eigenstates $|\uparrow \uparrow \uparrow\rangle$ and $|\downarrow \downarrow \downarrow\rangle$ of the $|\mathrm{GHZ}\rangle$ state. The analytical solution to the two coefficients is subject to a common normalizing factor and their ratio can be compactly written as

$$
\rho=\frac{c_{0}}{c_{3}}=\frac{6 \sqrt{6} g^{3} \mathfrak{f}^{3 / 2}}{\varepsilon(\varepsilon-\Delta \omega)(\varepsilon-2 \Delta \omega)-(11 \varepsilon-6 \Delta \omega) g^{2} \mathfrak{f}}
$$

By taking the derivative $d \rho / d \mathfrak{f}$, one finds the extrema of the ratio at

$$
\mathfrak{f}_{*}=\frac{3 \varepsilon(\varepsilon-\Delta \omega)(\varepsilon-2 \Delta \omega)}{11 \varepsilon-6 \Delta \omega}
$$

which is neither 0 nor 1 . Checking further that $d^{2} \rho /\left.d \mathfrak{f}^{2}\right|_{\mathfrak{f}_{*}}<0$, one finds $c_{0}$ would increase under inhomogeneous coupling, favoring $|\mathrm{GHZ}\rangle$ state generation. Substituting Eq. (8) into Eq. (2), one has the equation $U_{4}(\cos \pi \ell)-12 \mathfrak{f}_{*}+7=0$, where $U_{4}$ is the fourth-order Chebyshev polynomial of the second kind. Since the polynomial has four zeros, ignoring the two zeros on the negative axis of the even function $\cos \pi \ell$ shows that the ratio $\rho$ admits two maximizing values of $\ell$.

Using the experimental data given in Ref. [10], the numerical solutions of $c_{0}$ and $c_{3}$ are plotted for the highest split state of $\varepsilon$ in Fig. 3 against the relative spacing $\ell$ on one axis and against the detuning $\Delta \omega$ on the other. We observe that at exact lattice-photon resonance or at positive detuning, the coefficient $c_{0}$ (for the all spin-up state) is close to zero, meaning the probability of triple-photon resonance is low. In the range of negative detuning, $c_{0}$ starts to increase while the $c_{3}$ (for the all spin-down state) correspondingly decreases and their variations over the relative qubit spacing $\ell$ intensify. The plot shows that $c_{0}$ obtains maximum ( $c_{3}$ correspondingly obtains minimum) at two values: $\ell \approx 0.3$ and $\ell \approx 0.7$, verifying our analytical predictions above. Being neither 0 nor 1 and symmetric about the middle point $\ell=0.5$, the maxima show that inhomogeneous coupling increases the probability of 


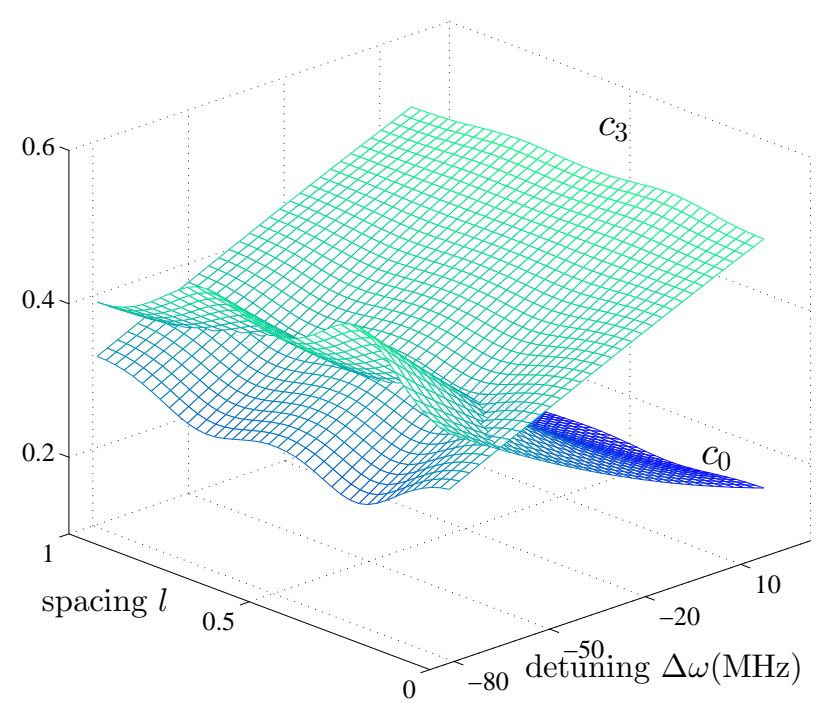

FIG. 3. (color online) The distribution of the normalized coefficients $c_{0}$ of the ground state and $c_{3}$ of the excited state versus the relative qubit spacing and the detuning for the clustered state $\left|u=\frac{3}{2}\right\rangle$.

multi-photon absorptions that lead to the generation of $|\mathrm{GHZ}\rangle$ state.

\section{MULTIPARTITE ENTANGLEMENT}

To further verify the results, we put the computed coefficients into two metrics of entanglement measures. One is the tripartite concurrence introduced by Carvalho et al. 22 that generalizes the original bipartite concurrence given by Wootters [21]. The other is the 3-tangle introduced by Coffman et al. [23]. Whereas 3-tangle quantifies only the three-party total non-separability, tripartite concurrence accounts for both partial and total nonseparabilities. In other words, when accounting the distributed entanglement among a 3-qubit quasi-lattice, tripartite concurrence does not distinguish the totally nonseparable $|\mathrm{GHZ}\rangle$ state and the partially non-separable $|W\rangle$ state. For example, the state $|\uparrow \downarrow \downarrow\rangle+|\downarrow \uparrow \downarrow\rangle=$ $(|\uparrow \downarrow\rangle+|\downarrow \uparrow\rangle) \otimes|\downarrow\rangle$ is partially non-separable and not totally non-separable. Hence, tripartite concurrence quantifies both types of non-separability as entanglement on an equal footing. 3-tangle, in contrast, is designed specifically to neglect the partial entanglement; it only measures the so-called residual entanglement that is left in a system after the partial entanglement are removed, i.e. it is non-zero only for $|\mathrm{GHZ}\rangle$ state [27, 31]. Here, by contrasting the two metrics over the same range of variation of a parameter, one is able to distinguish the indications of $|\mathrm{GHZ}\rangle$ state and $|W\rangle$ state in a quantum system.

Figure 4(a) plots the tripartite concurrence as a function of $\ell$ over the range $(0,1)$ for three detuning values of $\Delta \omega$. Figure 4 (b) plots 3-tangle against $\ell$ for the same three detunings. Other parameters are set to the same

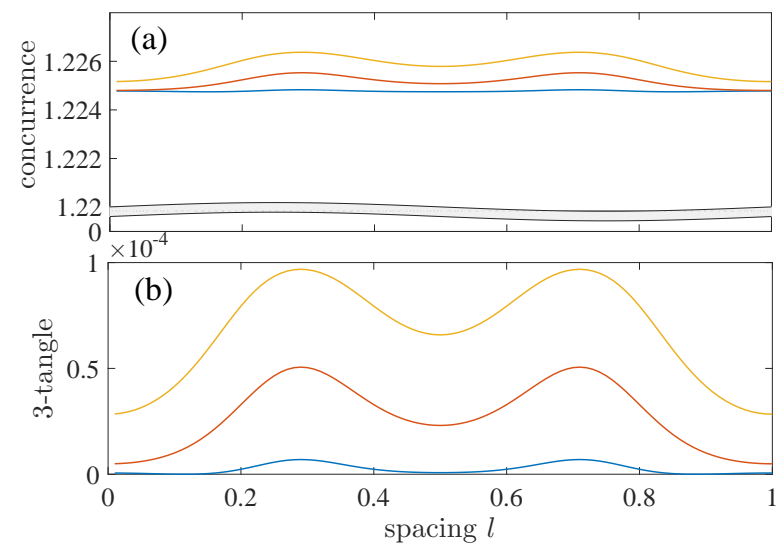

FIG. 4. (color online) Tripartite entanglements measured in two metrics: (a) the tripartite concurrence and (b) the 3tangle, at three values of detuning $\Delta \omega=-66 \mathrm{MHz}$ (yellow), $\Delta \omega=-92 \mathrm{MHz}$ (orange), and $\Delta \omega=-117 \mathrm{Mhz}$ (blue). In (a), the variation of the concurrence over $\ell$ is small compared to its average. We break the vertical axis to magnify the scope of variation.

values of those used in Fig. 3. For the three values of detunings, the concurrences are all close to the maximum attainable value of 1.25 , showing that the resonance between the quasi-lattice and the resonator photon generates highly entangled states among the qubits. It is particularly noticeable that the concurrence peaks at the same values of $\ell$ as those for the coefficient $c_{0}$, showing that multi-photon absorptions facilitated by inhomogeneous coupling also assist entanglement generation. However, the assistance is not large. For example, at $\Delta \omega=-92 \mathrm{MHz}$, the increase at the $\ell=0.3$ peak from $\ell=0$ is about $0.06 \%$.

3 -tangle demonstrate similar trends in variation over $\ell$ and peaks at the same points, even though its mean retains a small value throughout. Nevertheless, the variation is much more apparent: at $\Delta \omega=-92 \mathrm{MHz}, 3$-tangle is close to zero when the quasi-lattice is homogeneous at the two ends of abscissa; whereas at the $\ell=0.3$ peak, the increase over the two ends is about 94-fold.

These variations coincide with our perceptions. First, multi-photon processes are probabilistically rarer than single-photon processes, prompting lesser generations of $|\mathrm{GHZ}\rangle$ state than $|W\rangle$ state in general and giving a much lower mean in 3-tangle than in tripartite concurrence. Second, as predicted from our analysis of the coefficients, inhomogeneous coupling exemplified by the peaking values at $\ell \approx 0.3$ and $\ell \approx 0.7$ facilitates entanglement in general. If one regards concurrence and 3-tangle commensurate, then the contribution of 3-tangle to the concurrence increase at its maximal points occupies only about five to seven percent, showing not just $|\mathrm{GHZ}\rangle$ state generation but overall entangled state generation is facilitated. Third, inhomogeneous coupling does not assist but rather impedes single-photon processes because inhomogeneity decreases the qubit-photon coupling strength on average 
from the maximal value $g$. Since the coupling strength corresponds to the transition rate of each qubit, the probability of exciting any one qubit among all in the quasilattice from $\left|\frac{3}{2},-\frac{3}{2}\right\rangle$ to $\left|\frac{3}{2},-\frac{1}{2}\right\rangle$ is lowered. Our computation verifies that the coefficient $c_{2}$ for $\left|\frac{3}{2},-\frac{1}{2}\right\rangle$ indeed dips at $\ell=0.3$ and 0.7 . Nevertheless, inhomogeneous coupling does facilitate double-photon and triple-photon resonances, increasing the entanglement for inverted $|\bar{W}\rangle$ state and $|\mathrm{GHZ}\rangle$ state, respectively, especially the latter. Tripartite concurrence, accounting for all types of entanglement, is consequently compensated and adversely peaks at these originally dipping positions, resulting in a rather flat shape on average.

\section{CONCLUSIONS AND DISCUSSIONS}

We have demonstrated the role of inhomogeneous coupling in elevating the generation of $|\mathrm{GHZ}\rangle$ type of entanglement in a quasi-lattice of qubits, The analysis conducted above is confined to stationary states that form the bases of the dressed lattice-resonator system and it requires the transformation in Eq. (6) to compute the entanglement for an arbitrary state $|\psi\rangle$. How the entanglement would approach the stationary measures along with the evolution of $|\psi(t)\rangle$ however remains unknown and require future studies. But, in the least, from our studies of resonator-mediated double-cavity systems 32. and currently of cavity-coupled two-qubit system, we expect that: (i) the entanglement between the resonator and any one qubit it directly couples to can be transferred to that between two indirectly coupled qubits; (ii) the Rabi oscillations of the qubits render the stationary measures computed here an envelop in the time domain within which the entanglement would oscillate; (iii) it requires a finite time for the entanglement envelop to approach a stationary value, analogous to how $N$ atoms would radiate after a finite delay in a superradiant state.

\section{ACKNOWLEDGMENTS}

The author thanks X. G. Wang, Q. Ai, S. Ashhab, and E. Lucero for stimulating discussion. The research is supported by FDCT of Macau under Grant 013/2013/A1, University of Macau under Grants MRG022/IH/2013/FST and MYRG2014-00052FST, and the National Natural Science Foundation of China under Grant No. 11404415.
[1] Clarke J. and Wilhelm F. K., Nature 453 (2008) 1031-42.

[2] Blais A. et al., Phys. Rev. A 69 (2004) 062320.

[3] You J.Q. and Nori F., Phys. Rev. B 68 (2003) 64509; ibid., Nature 474 (2011) 589.

[4] Ashhab S. et al., New J. Phys. 11 (2009) 023030.

[5] André S. et al., Phys. Scr. 2009 (2009) 014016.

[6] Ian H., Liu Y.X. and Nori F., Phys. Rev. A 81 (2010) 063823.

[7] Joo J. et al., Phys. Rev. Lett. 105 (2010) 073601.

[8] Sun H.-C. et al., Phys. Rev. A 89 (2014) 063822.

[9] Castellanos-Beltran M. A. and Lehnert K. W., Appl. Phys. Lett. 91 (2007) 083509.

[10] Fink J. M. et al., Phys. Rev. Lett. 103 (2009) 083601.

[11] Macha P., et al., Nat. Commun. 5 (2014) 5146.

[12] Mlynek J. A. et al., Phys. Rev. A 86 (2012) 053838.

[13] Filipp S., et al., Phys. Rev. A 84 (2011) 061805.

[14] Mlynek J. A., Abdumalikov A. A., Eichler C. and Wallraff A., Nat. Commun. 5 (2014) 5186.

[15] Feng M. et al., Nat. Commun. 6 (2015) 7111.

[16] Colombe Y. et al., Nature 450 (2007) 272.
[17] Ian H. et al., Phys. Rev. A 78 (2008) 013824.

[18] Hunger D. et al., Phys. Rev. Lett. 104 (2010) 143002.

[19] Lucero E. et al., Nat. Phys. 8 (2012) 719.

[20] Ian H., Liu Y.X. and Nori F., Phys. Rev. A 85 (2012) 053833.

[21] Wootters W. K., Phys. Rev. Lett. 80 (1998) 2245.

[22] Carvalho A. R. R. et al., Phys. Rev. Lett. 93 (2004) 230501.

[23] Coffman V., Kundu J. and Wootters W. K., Phys. Rev. A 61 (2000) 052306.

[24] Wei. L. F. et al., Phys. Rev. Lett. 96 (2006) 246803.

[25] Matsuo S. et al., J. Phys. Soc. Jpn. 76 (2007) 54802.

[26] Zhang J. et al., Phys. Rev. A 79 (2009) 52308.

[27] Dür W., Vidal G. and Cirac J. I., Phys. Rev. A 62 (2000) 062314.

[28] Bennett C. H. et al., Phys. Rev. A 63 (2000) 012307.

[29] López C. E. et al., Phys. Rev. A 85 (2012) 032319.

[30] Ian H. and Liu Y.X., Phys. Rev. A 89 (2014) 043804.

[31] Lohmayer R. et al., Phys. Rev. Lett. 97 (2006) 260502.

[32] Huan T. et al., Phys. Rev. A 92 (2015) 022301. 